\title{
Dose Reduction and Dose Management in Computed Tomography - State of the Art
}

\section{Dosisreduktion und Dosismanagement in der Computertomografie - Aktueller Stand}

\author{
Authors \\ Dominik Zinsser ${ }^{1}$, Roy Marcus ${ }^{1}$, Ahmed E. Othman ${ }^{1}$, Fabian Bamberg ${ }^{1}$, Konstantin Nikolaou ${ }^{1}$, Thomas Flohr ${ }^{2}$, \\ Mike Notohamiprodjo ${ }^{1}$
}

Affiliation

1 Department of Diagnostic and Interventional Radiology, University Hospital Tübingen, Germany

2 CTE PA, Siemens Medical Solutions, Forchheim, Germany

Key words

$\mathrm{CT}$, diagnostic radiology, dose reduction, dose management

received 23.01.2017

accepted 31.12.2017

\section{Bibliography}

DOI https://doi.org/10.1055/s-0044-101261

Published online: 13.3 .2018

Fortschr Röntgenstr 2018; 190: 531-541

(c) Georg Thieme Verlag KG, Stuttgart · New York

ISSN 1438-9029

\section{Correspondence}

\section{Dominik Zinsser}

Department of Diagnostic and Interventional Radiology, University Hospital Tübingen, Hoppe-Seyler-Straße 3,

72076 Tübingen, Germany

Tel.: ++49/7071/2986677

dominik.zinsser@med.uni-tuebingen.de

\section{ABSTRACT}

Background For years, the number of performed CT examinations has been rising. At the same time, computed tomography became more dose efficient. The aim of this article is to give an overview about the state of the art in dose reduction in CT and to highlight currently available tools in dose management.

Method By performing a literature research on Pubmed regarding dose reduction in $\mathrm{CT}$, relevant articles were identified and analyzed.

Results and Conclusion Technical innovations with individual adaptation of tube current and voltage as well as iterative image reconstruction enable a considerable dose reduction with preserved image quality. At the same time, dedicated software tools are able to handle huge amounts of data and allow to optimize existing examination protocols.

\section{Key points}

- CT examinations are increasingly performed and contribute considerably to non-natural radiation exposure.

- A correct indication is crucial for each CT examination.

- The examination protocol has to be tailored to the medical question and patient.

- Multiple technical innovations enable considerable dose reduction with constant image quality.

- Dose management with dedicated software tools gains importance.

\section{Citation Format}

- Zinsser D, Marcus R, Othman AE et al. Dose reduction and dose management in computed tomography - State of the art. Fortschr Röntgenstr 2018; 190: 531-541

\section{ZUSAMMENFASSUNG}

Hintergrund Die Zahl der durchgeführten CT-Untersuchungen steigt seit Jahren an. Zugleich ist die Computertomografie dosiseffizienter geworden. Das Ziel dieses Artikels ist es, einen Überblick über den aktuellen Stand der Dosisreduktion im CT zu geben und derzeit verfügbare Werkzeuge zum Dosismanagement vorzustellen.

Methode Mittels einer Literatursuche bei Pubmed wurden relevante Artikel, die sich mit der Dosisreduktion in der CT beschäftigen, identifiziert und ausgewertet.

Ergebnisse und Schlussfolgerung Durch technische Neuerungen mit individueller Anpassung von Röhrenstrom und -spannung sowie iterativer Bildrekonstruktion ist eine deutliche Dosisreduktion bei erhaltener Bildqualität möglich. Zugleich erlauben entsprechende Softwaretools eine weitere Optimierung bestehender Untersuchungsprotokolle, wobei auch große Datenmengen mit wenig Aufwand verarbeitet werden können. 


\section{Introduction}

In recent decades computed tomography has increased in importance compared to other imaging methods. According to United States data, compared to the early 1980 s, in 2006 the number of CT examinations performed annually increased from about 3.3 to about 67 million. At the same time, the average per capita radiation exposure in the US caused by all medical procedures has increased sixfold from about $0.5 \mathrm{mSv}$ in 1980 to about $3 \mathrm{mSv}$ in 2006 , which is roughly comparable to the natural annual per capita radiation exposure. This is primarily due to increasing CT examination frequency, which accounts for only about $17 \%$ of all imaging examinations using ionizing radiation, but accounts for $49 \%$ of total radiation exposure [1]. Data from Germany likewise confirm this trend. According to the German Federal Office for Radiation Protection, the share of computed tomography in the collective effective dose caused by the medical application of X-rays in Germany was $65 \%$ in 2014, although a CT scan was performed in only $9 \%$ of all examinations [2]. At the same time, the incidence of CT examinations in Germany increased by about $40 \%$ between 2007 and 2014, leading to an increase in mean effective dose per inhabitant of about 1.4 to $1.6 \mathrm{mSv}$ caused by $\mathrm{X}$-ray applications [3]. Consequently, CT today represents the largest single source of non-natural exposure to the population, especially in developed countries.

\section{Why is dose reduction important?}

In view of the increased per capita radiation exposure from CT examinations, there are fears that these could lead to an increase in radiation-induced cancers [4-6]. On the other hand, it is believed that there is currently no evidence of adverse effects on human health by exposures comparable to natural radiation exposure in the range of an effective dose up to $100 \mathrm{mSv}$. The occasionally employed method of deriving an absolute number of additional cancers from the risk involved in such exposures and the frequency of examinations is therefore also criticized as speculative [7-10]. At the same time, however, there is also no safe threshold value, compliance with which can safely rule out damage by radiation exposure with absolute certainty. It should also be borne in mind that, for example, patients with chronic conditions may be exposed to cumulative doses of $100 \mathrm{mSv}$ or more due to repeated examinations. Therefore, the decision regarding a CT examination should always be preceded by a consideration process such as required by the $\mathrm{X}$-ray Ordinance or the Radiation Protection Act, which comes into force in 2018 [11, 12]. Ultimately, the benefits of a clinically-indicated CT outweigh the potential risk, provided that the applied radiation dose is appropriate to the issue as well as other factors such as the patient's age, pre-existing examinations and the suitability or availability of alternative examination methods are considered [10]. Conversely, avoiding a CT scan that is actually indicated may pose a risk since significant diagnoses can be overlooked [13].

Despite the lack of uniform data, considering the potential for harm from low-dose ionizing radiation, the ALARA (As Low As Reasonable Achievable) principle should be followed, and examinations should always be performed with the lowest dose possible to provide image quality that is sufficient to provide required diagnostic information $[10,13]$. In addition, the expected benefit must outweigh the risk, that is, the examination should be justified by the indication. Dose reduction alone is not an end in itself. A supposedly low-dose examination which does not provide sufficient image quality to provide a diagnosis harms the patient without providing a benefit.

The aim of this article is to provide an overview of common techniques used for dose reduction in computed tomography without any claim to completeness; reference should be made to further relevant literature, with numerous recent studies providing detailed insights into current developments in this field of medical imaging $[2,3,10,14-26]$.

\section{Dosimetry in Computed Tomography}

\section{CTDI and DLP}

The Computed Tomography Dose Index (CTDI) was designed to determine radiation emission during a CT scan and is based on measuring the absorbed dose in Plexiglas phantoms 16 or $32 \mathrm{~cm}$ in diameter to represent the X-ray attenuation of a human head and abdomen. The CTDI is a measure of the energy dose deposited in a single layer. The advent of spiral CT gave rise to the CT Volume Dose Index $\left(\mathrm{CTDI}_{\mathrm{vol}}\right)$ which is equivalent to the quotient of CTDI and pitch factor, reflecting the influence of the table pitch during the examination $[27,28]$. A pitch factor of 0.5 , for example, results from the overlapping scan in a dose increase by a factor of 2, which is correctly represented by the CTDI ${ }_{\text {vol }}$. The product of $\mathrm{CTDI}_{\mathrm{vol}}$ and the length of the scan volume is the dose-length product (DLP) as a measure of the total absorbed dose during the $C T$. It should be noted that the exposed volume exceeds the imaged body region in modern multi-line spiral CT scanners (over-beaming or over-ranging), with the associated increase in DLP dependent on the chosen collimation and pitch [29]. Generally both parameters are displayed on the control console during planning and after $\mathrm{CT}$, and documented in the dose protocol as well as the structured DICOM report. However, these merely represent the radiation dose delivered by the CT device, whereas the patient dose is a variable derived based on this and dependent on the mass and thus the stature of the patient [30-32].

\section{SSDE}

The concept of Size-Specific Dose Estimates (SSDE) has been developed by the American Association of Physicists in Medicine to overcome the drawback of absent reference of CTDI or DLP to the individual patient. In contrast to the aforementioned measured variables, the radiation emitted by the CT unit is set in relation to the patient size by multiplying the respective $C \mathrm{CDI}_{\mathrm{vol}}$ for calculating the SSDE by a factor corresponding to the estimated diameter of the patient [33]. As a further development of this concept, the use of the water-equivalent diameter has been proposed (equivalent to the diameter of a water-filled cylinder with the same attenuation as the patient), allowing a more accurate estimate of the patient's radiation exposure [34]. 


\section{Dose-determining Factors - Patient and Technique}

The radiation dose necessary for a sufficient image quality in the context of a CT examination depends on several factors, which are determined both by the patient as well as the technical parameters used.

\section{Patient factors}

The clinical issue, the patient's stature as well as position on the examination table significantly influence the radiation dose necessary for the CT scan.

A higher or lower radiation exposure is sufficient depending on the clinical issue ( $\triangleright$ Fig. 1). Thus, unlike the assessment of solid organs such as the liver in the examination of the lung parenchyma, or looking for urinary stones in which a high image noise level is acceptable due to the high environmental contrast of these structures; such noise is clearly less visible as a result of the typically wide image window selected (>1000 HU) [35], thus allowing reduced radiation exposure. Another example is the imaging of ventriculoperitoneal shunts, which can also be done using lowdose CT [14, 36, 37].

An important factor for the entire radiation exposure apart from the technical parameters is the extent of the body region of the patient to be examined for diagnostic purposes. Therefore the examination should be limited to the critical region; for example, to characterize adrenal lesions, the entire abdomen should not be imaged which would mean an additional but unjustified dose of radiation in this case. Likewise, repeated acquisitions in different phases in contrast-enhanced examinations are each associated with an additional radiation exposure. If this is absolutely necessary, for example, to clarify indistinct liver lesions, other examination modalities such as magnetic resonance imaging or contrastenhanced sonography may be used instead of CT [13].

Patient stature is important for the radiation dose in that a leaner patient requires less radiation dose for a given image noise and image quality compared to a heavier patient [28]. Conversely, if the scan parameters are not adapted, examining a bulky patient will result in increased image noise and possibly poorer image quality [38]. Accordingly, the examination protocol must be tailored to the particular patient.

Table positioning plays a major role here, since automatic modulation of tube current and voltage (see following sections) is based on the attenuation profile of the patient derived from the planning image. Positioning not centered on the middle of the body simulates a too large or small patient diameter due to the changed distance to the tube and detector and may be accompanied by excessive or low radiation exposure, which also affects the image quality ( $\triangleright$ Fig. 2) [39-41]. A study of an anthropomorphic thoracic phantom revealed a deviation of the radiation dose of -23 and +38 percent, respectively, when using a p. a. planning image for a table shifted $6 \mathrm{~cm}$ higher or lower than the correctly centered table position. In addition, it was shown that in clinical practice many CT examinations are carried out at a table height that is too low and therefore involve an unnecessarily high radiation exposure [40]. Correspondingly, to control proper cen-

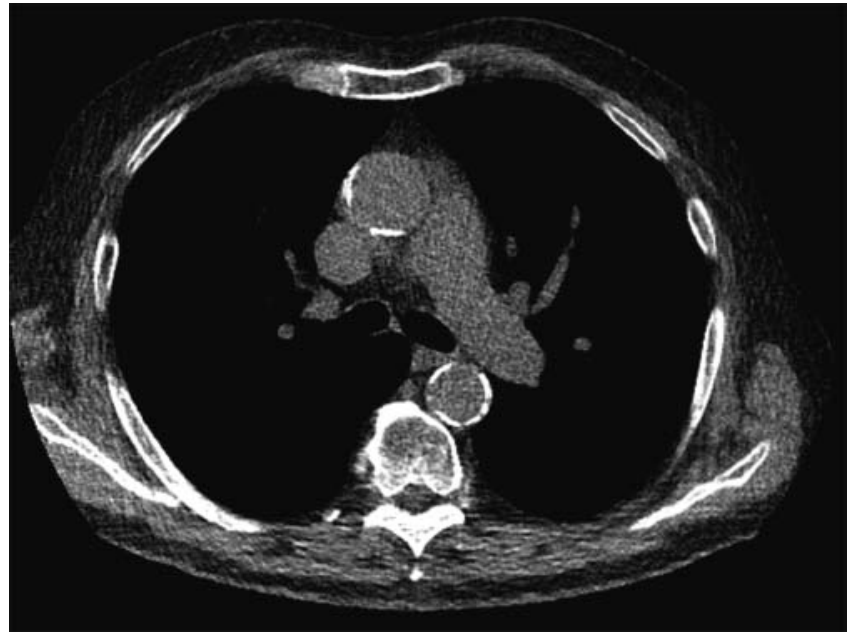

- Fig. 1 Low-dose CT of a patient before surgical replacement of the aortic valve for depiction of aortal calcifications; these are well evaluable, as well as the aortic diameter (CTDI ${ }_{\text {vol }} 0.42 \mathrm{mGy}$, DLP $14.1 \mathrm{mGy}^{*} \mathrm{~cm}$ ). The examination was performed using a Siemens SOMATOM Force.

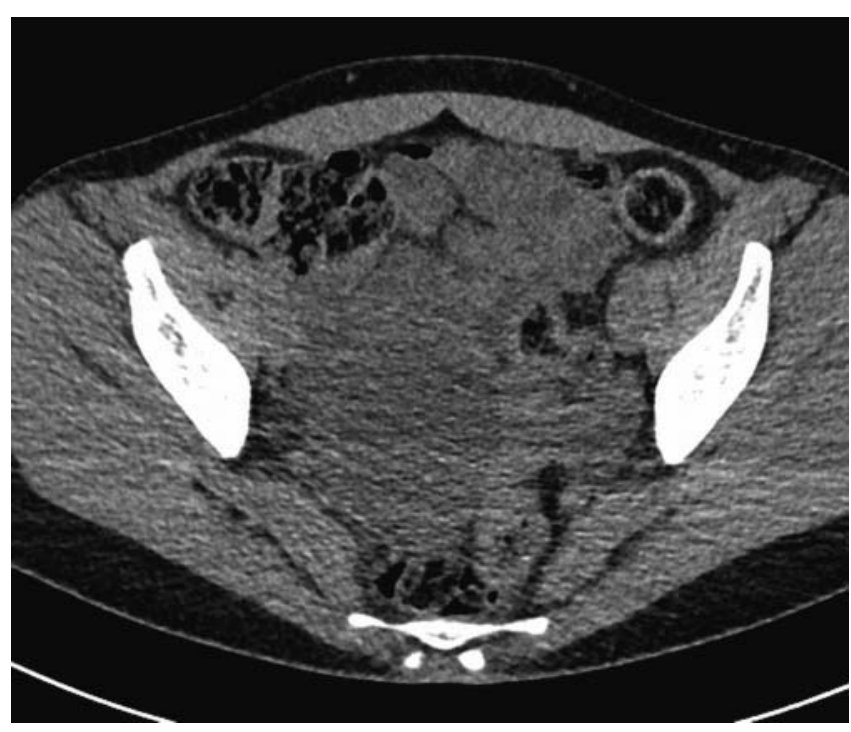

- Fig. 2 CT-examination of a female patient with suspected renal colic on the right side using automatic exposure control. Due to the not correctly centered examination table, the examination was performed with inadequate low radiation dose (CTDI $1.7 \mathrm{mGy}$, average $\mathrm{CTDI}_{\mathrm{vol}}$ at this scanner for this objective $3.4 \mathrm{mGy}$ ) resulting in high image noise despite using iterative reconstruction (ADMIRE 2). Therefore, the image quality was considered insufficient to detect small ureteral calculi.

tering, if necessary, an additional lateral planning image should be produced which allows the evaluation of table height [42], since the resulting minimal additional radiation dose appears justifiable. Another alternative is to acquire only a lateral planning image; thus, a study of low-dose CT imaging of the thorax demonstrated that this procedure is most efficient with respect to radiation hygiene [43]. It should be noted, however, that a repetition of the planning image may be necessary if faulty table 
positioning is revealed. Therefore, care should be taken to ensure the correct table height using the lateral laser marking when the patient is being positioned prior to examination.

\section{Technical Factors}

Tube current The photon flux generated by the X-ray tube is proportional to the tube current, so a $50 \%$ reduced tube current is equivalent to a $50 \%$ lower radiation dose. If the examination subject is unchanged, this dose reduction by a factor of 2 is accompanied by an $\sqrt{2}$-fold increase in image noise, or $41 \%$ [44]. The tube current can only be reduced to a limited extent since otherwise the proportion of photons penetrating the patient and contributing to the image information would be too limited. The converse idea that a higher tube current would always result in a subjectively better picture quality is incorrect, since the patient's constitution is also important here. For example, in the assessment of abdominal CT in obese patients, higher image noise is acceptable than in lean patients because the increased abdominal fat tissue provides a better contrast-to-noise ratio $[45,46]$.

Depending on the manufacturer, tube current is expressed either directly in milliamps $(\mathrm{mA})$ or in terms of the product of tube current and exposure time in mAs [35]. This can be adapted either manually or automatically to the patient's stature. The latter technique is one of the longest available dose reduction tools for CT and consists of two components that are typically used in parallel in current devices [45-47].

1. Modulation along the patient's longitudinal axis (z-axis modulation) is based on the varying attenuation of the radiation depending on the body region. For example, a higher radiation dose is required to examine the pelvis than for the predominantly air-filled thorax. Based on the pre-CT planning image, the attenuation of different body regions is estimated and the tube current adjusted in relation to the table pitch ( $\triangleright$ Fig. 3).

2. In contrast, modulation along the patient's transverse axis ( $x$-y axis modulation) is based on the fact that the human body usually has the shape of a horizontal ellipse with a greater transverse than sagittal diameter. Since image noise is largely determined by the transversal projections with high attenuation, the tube current in the sagittal projections can be significantly reduced and adjusted during each rotation related to the angle of the tube to the patient. The means of modulation is not the same for all manufacturers. Whereas, for example, devices made by Siemens perform permanent adaptation based on previously acquired projections offset by 180 degrees, GE dispenses with such real-time feedback, instead applying an undulating modulation similar to a sinusoidal curve ( $\vee$ Fig. 3 ).

Likewise, the algorithms used to adjust the tube current modulation are also manufacturer-dependent $[45,48]$. Siemens and Phillips rely on the parameters stored for a standard patient or a reference study to determine the intensity of the tube current modulation. These are selected by the user in such a way that an image quality sufficient for diagnosis is achieved (see also "Patient factors"). Instead of aiming for constant image noise regardless of the patient's stature, which would require a doubling of the applied dose even with a patient diameter that is about $4 \mathrm{~cm}$ lar-

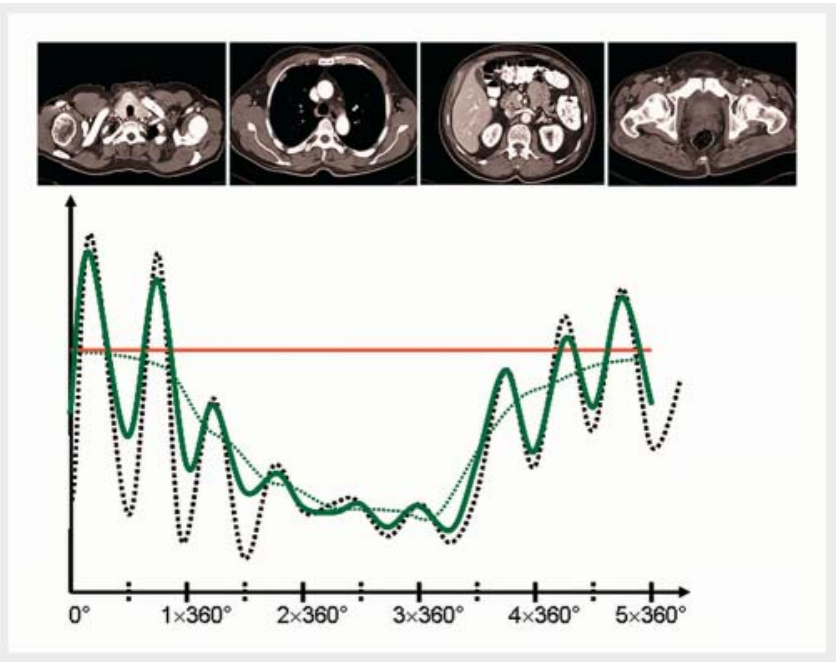

- Fig. 3 Depiction of tube current modulation along the patient's longitudinal axis (z-axis-modulation) as well as along the patient's transversal axis ( $\mathrm{x}$-y-axis-modulation) which shows the use of a higher current at body parts with higher attenuation (e. g. pelvis) and vice versa (e. g. thorax). At the same time, the tube current is modulated according to the angular tube position (higher tube current for transversal projections, lower tube current for sagittal projections); horizontal axis: table position; vertical axis: attenuation and tube current; red line: constant tube current; green line: tube current with activated automatic exposure control; dashed lines: attenuation.

ger, the modulation is adapted to examine obese patients with a lower dose and/or leaner patients with a higher dose that would be required to maintain constant image noise. The background of this procedure is based on the fact already mentioned above that in obese persons a higher image noise is considered tolerable, whereas in slim patients lower noise is desired. In addition, the intensity of the modulation can be influenced by the manufacturer, for example, Siemens allows graduation in five levels (very weak - weak - average - strong - very strong) [15]. GE and Toshiba, on the other hand, try to maintain constant image noise, regardless of body region and patient constitution. In order to achieve a proper contrast-to-noise ratio on equipment made by these manufacturers relevant to the patient's stature, either the desired intensity of the image noise can be varied according to the patient's size and weight, or minimum and maximum $\mathrm{mA}$ values can be used to ensure that slender persons are not examined with too low a dose or adipose persons receive too high a dose of radiation [46].

As a function of the body region examined, study protocols with tube current modulation versus fixed current-time product protocols have reported dose reduction of $20-68 \%$ without reducing the diagnostic utility, although overall higher image noise was reported, this was more homogeneous across the entire examination volume $[48,49]$.

Tube voltage Although the radiation emitted by an X-ray tube is the result of the interaction of tube voltage and current, and both parameters are typically not isolated from one another, for didactic reasons, sole consideration of tube voltage appears to be useful, since, unlike tube current, the relationship between 
tube voltage and emitted radiation is more complex, because the behavior is not linear, but approximately proportional to the square of the tube voltage. Presuming a constant current flow, the tube voltage is increased from 120 to $140 \mathrm{kV}$ thus providing a $50 \%$ higher dose, whereas a reduction from 120 to 100 or even $80 \mathrm{kV}$ provides a dose that is about 33 or $65 \%$ lower $[46,50]$. Adaptation of the tube voltage to the patient's stature and the medical issue thus offers high potential for dose reduction, although it should be noted that changing the contrast behavior is mainly related to the use of iodine-based contrast media.

The lower energy of the photons emitted at reduced voltage must be compensated for by a higher tube current in order to provide sufficient image information, since in this case the relative proportion of low-energy radiation increases, which is preferably absorbed in the patient and thus leads to an increase in dose without contributing to the image information. This is particularly true of adipose patients, so that a reduction of tube voltage can result in a higher dose of radiation. In addition, the CT unit may not have the necessary power reserves to adapt the tube current to examine obese patients with reduced tube voltage [50].

With lower tube voltage and the associated approximation of the K-edge of the iodine at $33.17 \mathrm{keV}$, the probability of interactions of the photons with iodine increases, thus attenuation by iodine-based contrast agents at lower tube voltage is stronger, and better contrast can be achieved at a lower tube voltage with iodine-based contrast agents. For this reason, a lower tube voltage is often useful in contrast-enhanced examinations, whereby the equivalent adjustment of the lower tube voltage can be replaced by a higher current flow, since the now lower-dose higher image noise is offset by better contrasting while still achieving a sufficient contrast-to-noise ratio [51]. In addition to CT angiography, this also applies to a lesser extent to other contrast-enhanced examinations; it should be kept in mind that when examining parenchymatous organs such as the liver, sufficient doses of radiation must be available for the detection of flaccid lesions. If this is not ensured at low tube voltages, a higher tube voltage scan may yield more favorable results. Other benefits of lower tube voltage include sparing contrast in patients with impaired renal function or achieving sufficient contrast in patients with poor venous conditions in whom only low flow rates are possible via peripheral access [50].

Adaptation of tube voltage can be performed manually based on parameters such as body weight or body-mass index. More sophisticated techniques for automatic selection of tube voltage have been developed recently, but they are not generally available. They can be combined with tube current modulation and are also based on the previously acquired planning image as well as the desired contrast-to-noise ratio for the respective medical issue. For example, the software offered by Siemens (CARE kV) allows graduation of the desired contrast-to-noise ratio in twelve steps in which higher levels purely retain the contrast-to-noise ratio, thus resulting in reduced tube voltage with lower radiation dose for the purpose of improved opacification while at the same time accepting higher image noise, as is needed for CT angiography (level 12 is provided for CT angiography). Lower levels do not reduce the radiation dose so strongly in order to improve contrast via transitioning to lower voltages while not substantially increas- ing image noise. This is advantageous for contrast-enhanced examinations of parenchymatous organs such as the liver (Level 7) Level 3 on the other hand maintains image noise during transition from one voltage level to another, and is used in native examinations [52]. Subsequently, various combinations of tube current and voltage are simulated on the basis of the established attenuation profile in order to determine the most dose-sparing combination for the respective examination $[16,51]$. Numerous studies have shown that good diagnostic image quality is possible together with reduced radiation exposure [18, 52, 53]. A recent article investigating the use of CARE kV for CT angiography prior to a planned catheter-supported aortic valve replacement additionally reported consistently high diagnostic image quality over a voltage range of $70-100 \mathrm{kV}$, based on objective and subjective criteria. On the other hand, in the case of adipose patients and the related higher tube voltages above $100 \mathrm{kV}$, there was poorer, yet still diagnostically usable image quality. Thus, the use of such dose reduction tools appears feasible regardless of the stature of the patient to be examined, since in the cited study adjustment of the quantity of contrast medium to the patient's weight was dispensed with, thereby allowing further improvement of contrast and with it image quality [17]. On the whole, this technique, based on the results of more than 100000 studies performed worldwide, allows for a CTRI vol -measured dose reduction of approximately $15 \%$, averaged over all body regions, compared to the practice of manually selecting tube voltage, but which also may be significantly higher, depending on the body region (about $56 \%$ in examinations of the petrous bone or $49 \%$ of pelvic/leg angiograms). Surprisingly, however, an increase in the average dose of $7 \%$ and $26 \%$, respectively, was found in examinations of the thoracic and lumbar spines and in detection of kidney and ureteral stones due to more frequent automatic selection of the maximum available tube voltage of $140 \mathrm{kV}$. However, according to the authors, it is unclear whether this increase is due to errors in the implementation and configuration of the software, or is a reflection of a general lack of suitability of the tool described for these types of CT examinations [16]. Likewise, in the area of pediatric radiology with respect to $\mathrm{CT}$ angiography and contrastenhanced examinations of the thorax and abdomen as well as of a phantom and patients, it was demonstrated that utilization of this tool compared to examination protocols with a fixed voltage of $120 \mathrm{kV}$, dose reduction of $27 \%$ was possible while maintaining image quality $[54,55]$. However, it should be noted here that in many cases a tube voltage of $120 \mathrm{kV}$ is less suitable for use in children, and that the advantage of automatic selection of tube voltage compared to fixed voltage values of $100 \mathrm{kV}$ or less should be significantly lower.

Pitch If all other scan parameters are kept constant, an isolated increase in the table pitch in the case of multi-slice CTs results in a proportionally lower examination dose, but also increased image noise [28]. Since Siemens and Phillips scanners perform automatic adaptation of the tube current to the pitch, keeping radiation dose, slice thickness and image noise constant independent of pitch, the above-described relationship between table pitch and radiation does not apply to these devices [35]. In this case a higher pitch is rather significant in order to reduce the examination time, thus avoiding movement artifacts. On the 
other hand, GE and Toshiba dispense with automatic tube current adaptation to pitch resulting in a higher pitch with a lower dose of radiation, but with higher noise in thin slices.

Image reconstruction Unlike previously discussed parameters, image reconstruction has no direct influence of patient dose. Compared to the otherwise typical filtered back projections (FBP), iterative reconstruction techniques developed in recent years have made it possible to achieve better image quality at the same dose, or comparable image quality at a lower dose especially with respect to image noise.

Filtered back projection is considered robust and fast, but requires a higher minimum applied dose; otherwise the quality of the examination can be severely limited by noise and artifacts. For example this can be observed with respect to adipose patients examined with insufficient radiation dose. An element of image reconstruction is a filtering process by means of convolution kernels which provide either higher image sharpness with emphasis on the edges of high-contrast objects (bone and lung) at the cost of higher image noise, or better assessment of low-contrast objects (parenchymatous organs) by reduced image noise with less sharpness [56, 57].

On the other hand, iterative reconstruction makes it possible to some extent to break through the relationship between increased image sharpness and raised image noise in filtered back projection while obtaining low image noise in the rather homogeneous areas of the image while preserving image sharpness, thereby reducing the radiation dose. This technique was already utilized for image reconstruction during the early years of $\mathrm{CT}$, but in the face of increasing quantities of data, it was shown to be impracticable, and was displaced by more rapid filtered back projection. With the development and availability of fast computers, however, the widespread clinical use of IR techniques has now become possible without much greater expenditure of time [56-58].

Stated simply, the principle of IR lies in the comparison of the measured $\mathrm{CT}$ raw data with simulated raw data to incrementally reduce image noise and artifacts. Generally, the first step consists of reconstruction using back projection. Then, using known device properties (scanner geometry, etc.), based on the CT image which now serves as a model for the examination object, a $\mathrm{CT}$ acquisition is simulated and simulated projection data is generated (forward projection). Measured and simulated raw data are compared in order to determine correction projections for the reconstruction of a "correction image", while at the same time nonlinear filter operations in the image and raw data spaces serve to reduce noise. This cycle is repeated several times until the desired image characteristics are achieved.

The iterative reconstruction techniques offered by different manufacturers differ in their complexity. Thus, the IR techniques commonly employed in clinical routine use less computationallydemanding and thus fewer time-consuming algorithms, since frequent back and forward projections are dispensed with. Instead, repeated filtering operations are performed to remove image noise and artifacts, which can occur at either the image or raw data level or at both levels. The frequency and with what filter strength these steps are performed can be incrementally graduated by the user with many of the commercially-available IR systems. IRIS by Sie- mens is an example of an iterative reconstruction algorithm that only applies on the image data level, whereas SAFIRE and ADMIRE (Siemens), AIDR 3 D (Toshiba), iDose 4 (Phillips), and ASIR-V (GE) are representatives of image-level and raw-data-level iterative reconstruction techniques [56-58].

On the other hand, statistical iterative reconstruction is based on the premise of statistical weighting of the raw data performed with each cycle of back and forward projection with the aim of allowing very noisy raw data to contribute less to the image, thus reducing image noise. An example of this is the VEO iterative reconstruction technique offered by GE. To increase image sharpness, such methods may also more accurately model the scanner geometry (size of detector pixels and focus in the X-ray source); this is called model-based iterative reconstruction (MBIR). The nomenclature used by manufacturers is not always the same. Thus, the algorithm offered by GE as ASIR (adaptive statistical iterative reconstruction) represents one of the first iterative reconstruction methods and is only somewhat comparable to the more recent statistical and model-based methods. Statistical iterative reconstructions and model-based iterative reconstructions require many cycles of back and forward projection, are therefore very computationally demanding and usually have long image reconstruction times, thus limiting their use in the clinical routine, although studies have demonstrated improved image quality during reconstruction of low-dose CT data [19]. However this could change with the employment of newer generations of statistically iterative reconstruction algorithms. A recently published study of the use of such prototypes for CT coronary angiography reports a reconstruction time of only five minutes - which appears justifiable for clinical application - with an accompanying reduced radiation dose and improved image quality [20].

A problem with the use of iterative reconstruction techniques can be the changed image impression, in particular with higher noise reduction, since more homogeneous surfaces can appear increasingly "plastic-like" and as though painted ( $\mathbf{F i g . 4 a - e )}$ [57]. Especially when introducing iterative reconstruction, it is therefore recommended to start at a lower level in order to allow the user to gradually adjust to the altered image impression.

Over the last few years numerous studies on the potential of IR dose-reduction techniques have been published which report with savings of approximately $20 \%$ to over $75 \%[21,59,60]$. However, one study has shown that too aggressive dose reduction, despite the application of IR, leads to a loss of low contrast resolution in the assessment of objects with limited environmental contrast. The authors of this study therefore advise against too aggressive dose reduction despite using IR, if for example, native skull examinations to rule out stroke, or contrast-enhanced liver examinations are performed to detect metastases, since sufficient contrast resolution even in the case of low contrast is critical to prevent overlooking e.g., lesions [61].

\section{Low-dose Simulation}

The feasibility and extent of dose reduction without diminishing image quality and diagnostic value must be reviewed prior to the clinical use of dose-reduced protocols. In this case low-dose simu- 

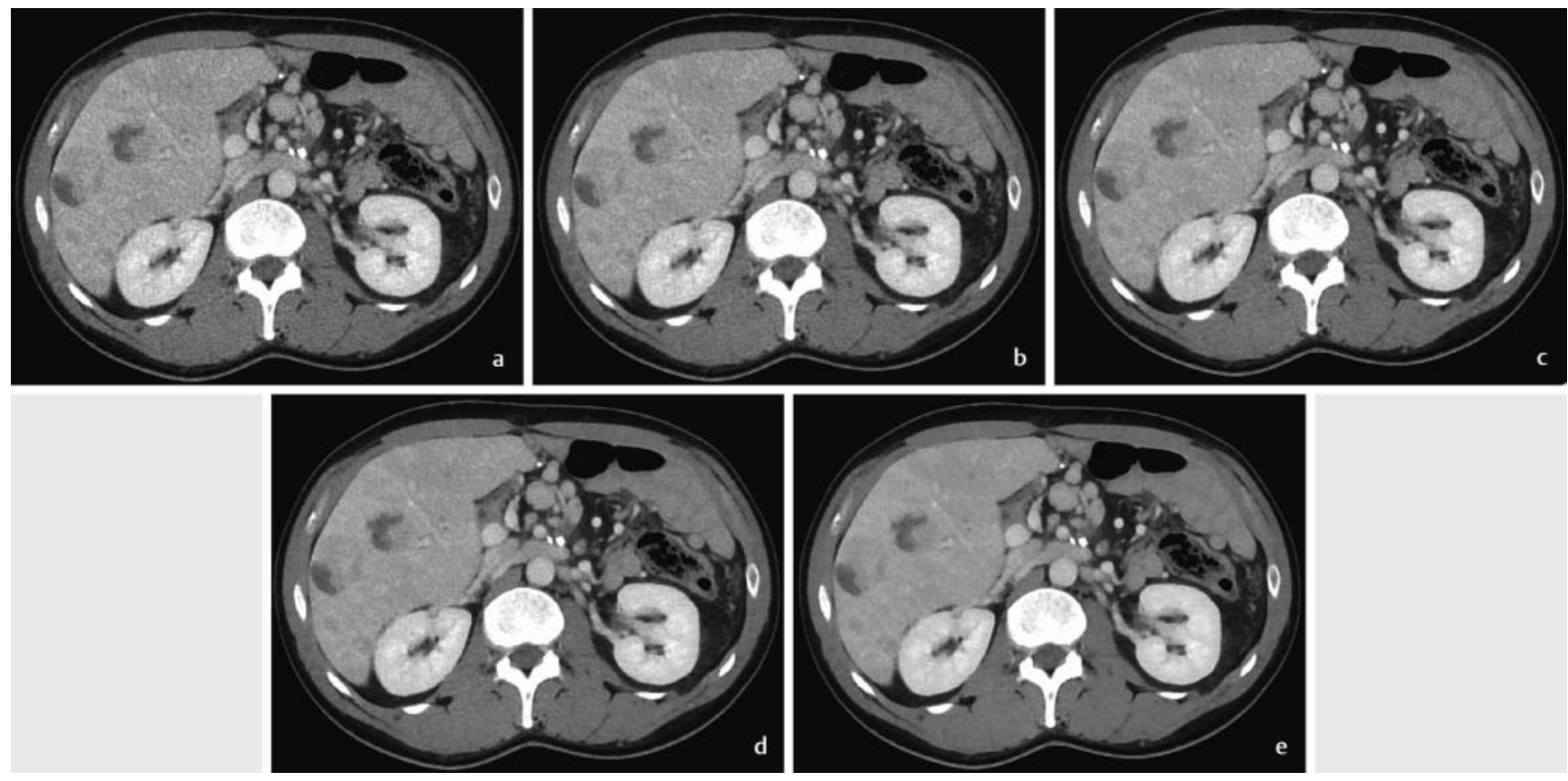

- Fig. 4 CT examination of a patient with hepatic and lymphogenous metastasized neuroendocrine tumor of the pancreas as example for the altered image impression with iterative reconstruction. Images were reconstructed using ADMIRE (Advanced Modeled Iterative Reconstruction, Siemens Healthineers) in five ascending degrees of iteration with window width of $350 \mathrm{HU}$ and centre of $50 \mathrm{HU}$. By decreasing image noise, homogenous areas as the liver parenchyma show lower contrast while the stranding of the perirenal adipose tissue looks more and more blurred when using high degrees of ADMIRE (a ADMIRE 1, b ADMIRE 2, c ADMIRE 3, d ADMIRE 4 and e ADMIRE 5).

lation can be useful since it allows retrospectively dose-reduced CT data by simulating CT examinations with reduced tube current by adding realistic image noise to existing original data [62]. This is being increasingly employed, for example in developing a cranial low-dose CT perfusion protocol [22, 23, 63, 64]. It was demonstrated that a dose reduction of up to $60 \%$ of the original standard dose does not result in image quality deterioration or diagnostic accuracy using this method.

\section{Dose Monitoring and Management}

In recent years computed tomography has continued to evolve and become more dose-efficient. This is also reflected in the most recently corrected diagnostic reference values of the Federal Office for Radiation Protection last July, which correspond to "the 75th percentile of a distribution of patient doses of different users" and are not to be understood as "ideally achievable values" [24]. Current equipment can achieve even lower doses than indicated in the diagnostic reference values. The possibilities of the state of the art are not always implemented in clinical practice as the situation in Germany recently demonstrated [65]. In order to meet the potential of sometimes complex technical innovations, an optimization process is required that requires an overview of where improvements are possible. Although a dose report is usually created for each CT and stored with the images in the PACS, manually sampling this information to identify particularly high dose examinations is time consuming and can only provide an incomplete picture. Another independent but no less important aspect of dose monitoring concerns the already mentioned problem of high cumulative doses of individual patients resulting from repeated examinations, which cannot be readily determined without suitable aids [25].

In the meantime several manufacturers have offered software tools for dose management, which allow the average dose of individual examination protocols to be determined on the basis of parameters such as CTDI, DLP, effective dose or SSDE and offer the potential to discover optimization possibilities, allowing other modalities to be included in addition to computed tomography.

- Table 1 contains an overview of dose management systems available on the market; refer to the corresponding publications for a detailed description [26, 66]. Based on the example of Radimetrics (Bayer) which we have used in our department, the following will describe typical capabilities of dose management programs. The user can set limits for examination protocols and receives a warning when these are exceeded. Frequent deviations are indicative of systematic errors when performing CT examinations which can be discovered and remedied. Likewise thresholds can be established for cumulative patient doses, and warnings can be generated if they are likely to be exceeded so that the affected patient can undergo a different suitable examination, as needed. Dose monitoring by means of automatically generated alarm messages based on limit values predefined by the user can thus occur both on the level of individual examination protocols as well as for individual patients [25]. Likewise, for example, the average dose requirement of various CT devices for the examination of certain body regions can be determined and compared in this way, so that e.g., younger patients can be examined using dosesparing equipment ( $\mathbf{F i g . 5}$ ) [67]. Large quantities of data can 


\begin{tabular}{|c|c|c|c|c|c|c|c|}
\hline & 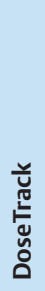 & 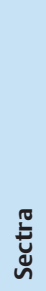 & 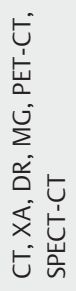 & $\frac{\bar{O}}{\mathrm{O}}$ & 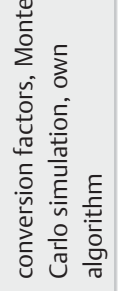 & $\stackrel{\tilde{y}}{\beth}$ & 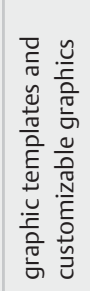 \\
\hline & 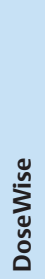 & $\frac{\text { 毫 }}{\frac{\bar{c}}{\alpha}}$ & 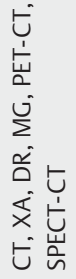 & $\begin{array}{l}\overline{\widetilde{U}} \\
\underline{0}\end{array}$ & 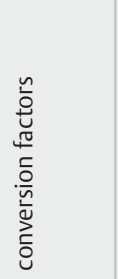 & $\stackrel{\Perp}{\nu}$ & 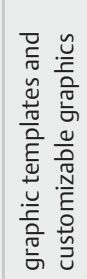 \\
\hline 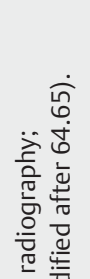 & $\begin{array}{l}\sum_{\grave{U}} \\
\text { 口े }\end{array}$ & 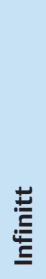 & 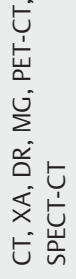 & 픙 & 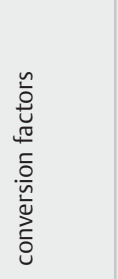 & $\stackrel{\tilde{\nu}}{\nu}$ & 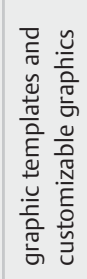 \\
\hline 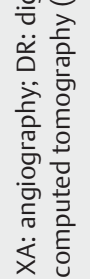 & 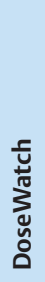 & 岕 & 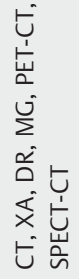 & $\begin{array}{l}\bar{\Xi} \\
\text { 。 }\end{array}$ & 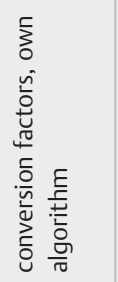 & $\stackrel{\tilde{\nu}}{\beth}$ & 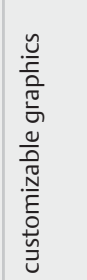 \\
\hline 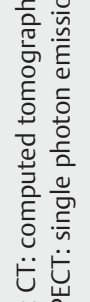 & 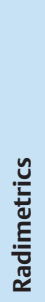 & స్ల & 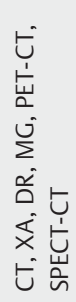 & 픔 & 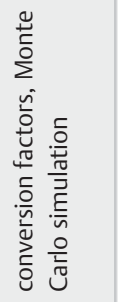 & $\stackrel{\check{\nu}}{\beth}$ & 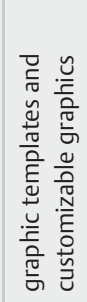 \\
\hline 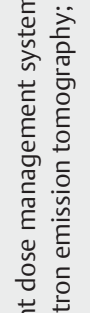 & 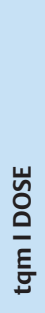 & $\frac{\pi}{2}$ & 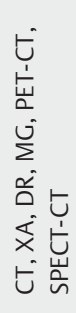 & 픙 & 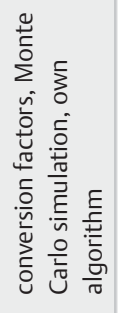 & 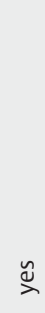 & 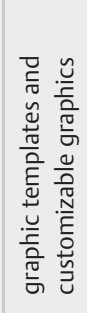 \\
\hline 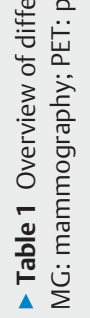 & $\begin{array}{l}\text { E } \\
\text { ָ }\end{array}$ & 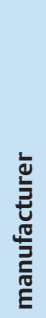 & 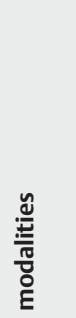 & 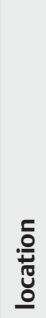 & 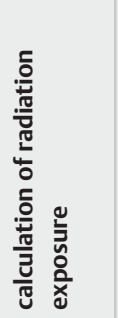 & 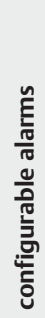 & 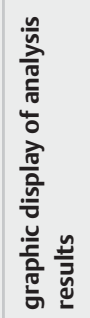 \\
\hline
\end{tabular}

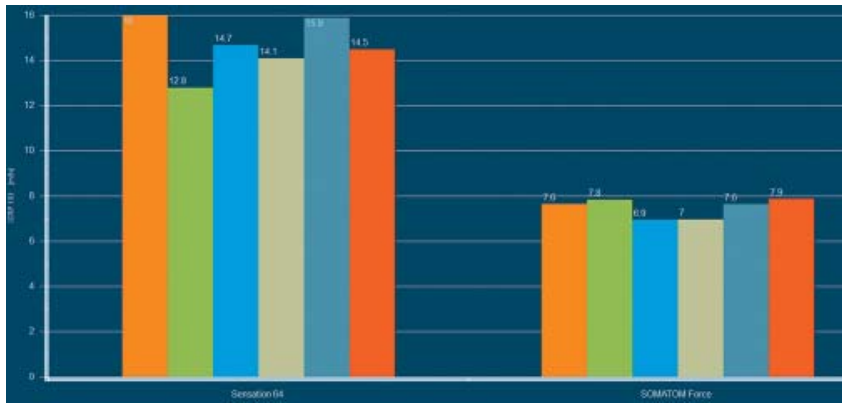

- Fig. 5 Bar chart comparing average effective dose for a standard abdominal CT examination over six consecutive months performed using an older 64-row-scanner without iterative reconstruction (Siemens Sensation 64, left) and a latest generation dual-sourcescanner with iterative reconstruction (Siemens Somatom Force, right). Due to the considerably lower radiation dose, we preferably perform this examination using the newer $\mathrm{CT}$-device which leads to a considerably higher number of examinations (Sensation 64: 63 examinations, Somatom Force: 483 examinations; not depicted). There is no systematic difference regarding the physical constitution of both examined patient collectives.

be processed with relatively little effort; we refer to a publication in the United States which analyzed data on the average dose of almost 200000 CT scans performed in one year on more than 80000 patients in five different clinics [68]. In addition, studies can be simulated to determine the effects of changing parameters such as scan length, tube voltage and current on the patient dose using stored phantoms and Monte Carlo simulations, which can be used, for example, for training in the training of radiological technical assistants.

The EURATOM Directive 2013/59 which is to be implemented in national law in 2018, provides for increased involvement of medical physics experts in $\mathrm{CT}$; their task is the "optimization of patient radiation protection [...] including the use and application of diagnostic reference values" [69]. The directive does not specify how such medical physics experts should perform this task. In view of the enormous amounts of data, the implementation of efficient monitoring and optimization only seems possible if tools such those presented here are used.

\section{Summary and Outlook}

In view of the increasing number of examinations and associated concerns about possible long-term consequences, numerous technical innovations have been introduced over the last few years which have made CT more dose-efficient. It is expected that this process will continue in the future. At the same time, the trend is towards increasing monitoring and optimization of the radiation dose applied to the patient. The challenge for radiology is to translate the possibilities that arise into clinical routine to provide patients with a good, yet at the same time, as harmless a diagnostic procedure as possible.

Since not all aspects of this topic can be presented in a single review article, we would refer readers interested in more in-depth knowledge to the additional literature listed in the appendix. 


\section{Conflict of Interest}

Fabian Bamberg: Unrestricted research grant and member Speakers Bureau Siemens Healthineers, unrestricted reserach grant and member Speakers Bureau Bayer. Ahmed Othman: Unrestricted research grant Bayer and member Speakers Bureau GE Healthcare. Thomas Flohr: Staff member Siemens Healthineers. The other authors declare that they have no conflict of interest.

\section{References}

[1] Mettler FA Jr, Bhargavan M, Faulkner K et al. Radiologic and nuclear medicine studies in the United States and worldwide: frequency, radiation dose, and comparison with other radiation sources-1950-2007. Radiology 2009; 253: 520-531

[2] Bundesamt für Strahlenschutz BfU, Naturschutz, Bau und Reaktorsicherheit. Umweltradioaktivität und Strahlenbelastung: Jahresbericht 2015. http://nbn-resolving.de/urn:nbn:de:0221-2017072814305 (last accessed on 31.08.2017)

[3] Nekolla EA, Schegerer AA, Griebel J et al. Frequency and doses of diagnostic and interventional Xray applications : Trends between 2007 and 2014. Der Radiologe 2017; 57: 555-562

[4] Berrington de Gonzalez A, Mahesh M, Kim KP et al. Projected cancer risks from computed tomographic scans performed in the United States in 2007. Archives of internal medicine 2009; 169: 2071-2077

[5] Smith-Bindman R, Lipson J, Marcus R et al. Radiation dose associated with common computed tomography examinations and the associated lifetime attributable risk of cancer. Archives of internal medicine 2009; 169: $2078-2086$

[6] Brenner DJ, Hall EJ. Computed tomography-an increasing source of radiation exposure. The New England journal of medicine 2007; 357: $2277-2284$

[7] Hendee WR. Policy statement of the International Organization for Medical Physics. Radiology 2013; 267: 326-327

[8] Protection ICoR: The 2007 Recommendations of the International Commission on Radiological Protection. ICRP publication 103. Annals of the ICRP 2007; 37: 1-332

[9] Hendee WR, O'Connor MK. Radiation risks of medical imaging: separating fact from fantasy. Radiology 2012; 264: 312-321

[10] McCollough CH. The Role of the Medical Physicist in Managing Radiation Dose and Communicating Risk in CT. Am J Roentgenol 2016; 206: $1241-1244$

[11] Verordnung über den Schutz vor Schäden durch Röntgenstrahlen (Röntgenverordnung - RöV). http://www.gesetze-im-internet.de/ r_v_1987/R\%C3\%B6V.pdf (last accessed on 31.08.2017)

[12] Gesetz zur Neuordnung des Rechts zum Schutz vor der schädlichen Wirkung ionisierender Strahlung. http://www.bmub.bund.de/fileadmin/ Daten_BMU/Download_PDF/Strahlenschutz/neuordnung_wirkung_ionisierender_strahlung.pdf (last accessed on 31.08.2017)

[13] Mayo-Smith WW, Hara AK, Mahesh M et al. How I do it: managing radiation dose in CT. Radiology 2014; 273: 657-672

[14] Afat S, Pjontek R, Hamou HA et al. Imaging of Ventriculoperitoneal Shunt Complications: Comparison of Whole Body Low-Dose Computed Tomography and Radiographic Shunt Series. Journal of computer assisted tomography 2016; 40: $991-996$

[15] Soderberg M. Overview, practical tips and potential pitfalls of using automatic exposure control in CT: Siemens CARE Dose 4D. Radiation protection dosimetry 2016; 169: 84-91

[16] Spearman JV, Schoepf UJ, Rottenkolber M et al. Effect of Automated Attenuation-based Tube Voltage Selection on Radiation Dose at CT: An Observational Study on a Global Scale. Radiology 2016; 279: 167 - 174
[17] Mangold S, De Cecco CN, Schoepf U] et al. CT angiography for planning transcatheter aortic valve replacement using automated tube voltage selection: Image quality and radiation exposure. European journal of radiology 2017; 86: $276-283$

[18] Mangold S, Wichmann JL, Schoepf UJ et al. Automated tube voltage selection for radiation dose and contrast medium reduction at coronary $\mathrm{CT}$ angiography using 3(rd) generation dual-source CT. European radiology 2016; 26: $3608-3616$

[19] Sun J, Yu T, Liu J et al. Image quality improvement using model-based iterative reconstruction in low dose chest $C T$ for children with necrotizing pneumonia. BMC medical imaging 2017; 17: 24

[20] Andre F, Fortner P, Vembar M et al. Improved image quality with simultaneously reduced radiation exposure: Knowledge-based iterative model reconstruction algorithms for coronary $\mathrm{CT}$ angiography in a clinical setting. Journal of cardiovascular computed tomography 2017; 11: 213-220

[21] Den HarderAM, Willemink M], De Ruiter QM et al. Dose reduction with iterative reconstruction for coronary $\mathrm{CT}$ angiography: a systematic review and meta-analysis. The British journal of radiology 2016; 89: 20150068

[22] Othman AE, Afat S, Brockmann MA et al. Radiation dose reduction in perfusion $C T$ imaging of the brain: A review of the literature. Journal of neuroradiology Journal de neuroradiologie 2016; 43: 1 - 5

[23] Othman AE, Brockmann C, Yang Z et al. Impact of image denoising on image quality, quantitative parameters and sensitivity of ultra-low-dose volume perfusion CT imaging. European radiology 2016; 26: 167-174

[24] Strahlenschutz Bf: Bekanntmachung der aktualisierten diagnostischen Referenzwerte für diagnostische und interventionelle Röntgenanwendungen. BAnz AT Bundesamt für Strahlenschutz 2016

[25] Parakh A, Kortesniemi M, Schindera ST. CT Radiation Dose Management: A Comprehensive Optimization Process for Improving Patient Safety. Radiology 2016; 280: 663-673

[26] Boos ], Meineke A, Bethge OT et al. Dose Monitoring in Radiology Departments: Status Quo and Future Perspectives. RoFo : Fortschritte auf dem Gebiete der Rontgenstrahlen und der Nuklearmedizin 2016; 188: $443-450$

[27] Kalra MK, Maher MM, Toth TL et al. Strategies for CT radiation dose optimization. Radiology 2004; 230: 619-628

[28] McNitt-Gray MF. AAPM/RSNA Physics Tutorial for Residents: Topics in $\mathrm{CT}$. Radiation dose in CT. Radiographics: a review publication of the Radiological Society of North America, Inc 2002; 22: 1541-1553

[29] Schilham A, van der Molen AJ, Prokop M et al. Overranging at multisection CT: an underestimated source of excess radiation exposure. Radiographics : a review publication of the Radiological Society of North America, Inc 2010; 30: 1057-1067

[30] McCollough CH, Leng S, Yu L et al. CT dose index and patient dose: they are not the same thing. Radiology 2011; 259: 311-316

[31] Huda W, Mettler FA. Volume CT dose index and dose-length product displayed during CT: what good are they? Radiology 2011; 258: 236-242

[32] Brink JA, Morin RL. Size-specific dose estimation for CT: how should it be used and what does it mean? Radiology 2012; 265: 666-668

[33] Medicine AAoPi. Size-specific dose estimates (SSDE) in pediatric and adult body CT Examinations: Report of AAPM Task Group 204. College Park: Md: American Association of Physicists in Medicine. 2011

[34] Medicine AAoPi. Use of Water Equivalent Diameter for Calculating Patient Size and Size-Specific Dose Estimates (SSDE) in CT: The Report of AAPM Task Group 220. College Park, Md: American Association of Physicists in Medicine. 2014

[35] Kalra MK, Sodickson AD, Mayo-Smith WW. CT Radiation: Key Concepts for Gentle and Wise Use. Radiographics : a review publication of the Radiological Society of North America, Inc 2015 35: 1706-1721

[36] Othman A, Hamou HA, Pjontek R et al. Evaluation of whole body UltralowDose $C T$ for the assessment of ventriculoperitoneal shunt complications: 
an experimental ex-vivo study in a swine model. European radiology 2015; 25: 2199-2204

[37] Othman AE, Afat S, Hamou HA et al. High-Pitch Low-Dose Whole-Body Computed Tomography for the Assessment of Ventriculoperitoneal Shunts in a Pediatric Patient Model: An Experimental Ex Vivo Study in Rabbits. Investigative radiology 2015; 50: 858-862

[38] Wilting JE, Zwartkruis A, van Leeuwen MS et al. A rational approach to dose reduction in $\mathrm{CT}$ : individualized scan protocols. European radiology 2001; 11: 2627-2632

[39] Li ], Udayasankar UK, Toth TL et al. Automatic patient centering for MDCT: effect on radiation dose. American journal of roentgenology 2007; 188: 547 - 552

[40] Kaasalainen T, Palmu K, Reijonen V et al. Effect of patient centering on patient dose and image noise in chest CT. American journal of roentgenology 2014; 203: 123 - 130

[41] Toth T, Ge Z, Daly MP. The influence of patient centering on CT dose and image noise. Medical physics 2007; 34: 3093 - 3101

[42] Lee CH, Goo JM, Ye HJ et al. Radiation dose modulation techniques in the multidetector CT era: from basics to practice. Radiographics : a review publication of the Radiological Society of North America, Inc 2008; 28 : $1451-1459$

[43] Bang DH, Lim D, Hwang WS et al. Lateral topography for reducing effective dose in low-dose chest CT. Am J Roentgenol American journal of roentgenology 2013; 200: 1294-1297

[44] Maldjian PD, Goldman AR. Reducing radiation dose in body CT: a primer on dose metrics and key CT technical parameters. American journal of roentgenology 2013; 200: 741 - 747

[45] McCollough CH, Bruesewitz MR, Kofler JM Jr. CT dose reduction and dose management tools: overview of available options. Radiographics : a review publication of the Radiological Society of North America, Inc 2006; 26: $503-512$

[46] Kaza RK, Platt JF, Goodsitt MM et al. Emerging techniques for dose optimization in abdominal CT. Radiographics : a review publication of the Radiological Society of North America, Inc 2014; 34: 4- 17

[47] Kalra MK, Maher MM, Toth TL et al. Techniques and applications of automatic tube current modulation for CT. Radiology 2004; 233: 649-657

[48] Soderberg M, Gunnarsson M. Automatic exposure control in computed tomography-an evaluation of systems from different manufacturers. Acta radiologica (Stockholm, Sweden : 1987) 2010; 51: 625-634

[49] Mulkens TH, Bellinck P, Baeyaert M et al. Use of an automatic exposure control mechanism for dose optimization in multi-detector row CT examinations: clinical evaluation. Radiology 2005; 237: 213-223

[50] Seyal AR, Arslanoglu A, Abboud SF et al. CT of the Abdomen with Reduced Tube Voltage in Adults: A Practical Approach. Radiographics : a review publication of the Radiological Society of North America, Inc 2015; 35: $1922-1939$

[51] Lira D, Padole A, Kalra MK et al. Tube potential and CT radiation dose optimization. American journal of roentgenology 2015; 204: W4-W10

[52] Gonzalez-Guindalini FD, Ferreira BotelhoMP, Tore HG et al. MDCT of chest, abdomen, and pelvis using attenuation-based automated tube voltage selection in combination with iterative reconstruction: an intrapatient study of radiation dose and image quality. American journal of roentgenology 2013; 201: 1075-1082
[53] Lee KH, Lee JM, Moon SK et al. Attenuation-based automatic tube voltage selection and tube current modulation for dose reduction at contrast-enhanced liver CT. Radiology 2012; 265: 437-447

[54] Siegel M], Ramirez-Giraldo JC, Hildebolt C et al. Automated low-kilovoltage selection in pediatric computed tomography angiography: phantom study evaluating effects on radiation dose and image quality. Investigative radiology 2013; 48: 584 - 589

[55] Siegel M], Hildebolt C, Bradley D. Effects of automated kilovoltage selection technology on contrast-enhanced pediatric CT and CT angiography. Radiology 2013; 268: 538 - 547

[56] Geyer LL, Schoepf UJ, Meinel FG et al. State of the Art: Iterative CT Reconstruction Techniques. Radiology 2015; 276: 339-357

[57] Willemink M], de Jong PA, Leiner T et al. Iterative reconstruction techniques for computed tomography Part 1: technical principles. European radiology 2013; 23: 1623-1631

[58] Beister M, Kolditz D, Kalender WA. Iterative reconstruction methods in X-ray CT. Physica medica : PM : an international journal devoted to the applications of physics to medicine and biology : official journal of the Italian Association of Biomedical Physics (AIFB) 2012; 28: 94 - 108

[59] Kalra MK, Woisetschlager M, Dahlstrom N et al. Radiation dose reduction with Sinogram Affirmed Iterative Reconstruction technique for abdominal computed tomography. Journal of computer assisted tomography 2012; 36: $339-346$

[60] Willemink M], Leiner T, de Jong PA et al. Iterative reconstruction techniques for computed tomography part 2 : initial results in dose reduction and image quality. European radiology 2013; 23: $1632-1642$

[61] McCollough CH, Yu L, Kofler JM et al. Degradation of CT Low-Contrast Spatial Resolution Due to the Use of Iterative Reconstruction and Reduced Dose Levels. Radiology 2015; 276: 499-506

[62] Won KimC, Kim JH. Realistic simulation of reduced-dose CT with noise modeling and sinogram synthesis using DICOM CT images. Medical physics 2014; 41: 011901

[63] Othman AE, Brockmann C, Yang Z et al. Effects of radiation dose reduction in Volume Perfusion CT imaging of acute ischemic stroke. European radiology 2015; 25: $3415-3422$

[64] Othman AE, Afat S, Brockmann C et al. Low-Dose Volume-Perfusion CT of the Brain: Effects of Radiation Dose Reduction on Performance of Perfusion CT Algorithms. Clinical neuroradiology 2015; 27: 311 - 318

[65] Schegerer AA, Nagel H-D, Stamm G et al. Current CT Practice in Germany: Results and Implications of a Nationwide Survey. European journal of radiology 2017; 90: 114-128

[66] Dosis-Management - Die wichtigsten Systeme im Überblick. http://www.radiologieforum.de/uploads/Magazine/epaper-Strada-2016/page41.html\#/40 (last accessed on 22.03. 2017)

[67] MacGregor K, Li I, Dowdell T et al. Identifying Institutional Diagnostic Reference Levels for CT with Radiation Dose Index Monitoring Software. Radiology 2015; 276: 507 - 517

[68] Smith-Bindman R, Moghadassi M, Wilson N et al. Radiation Doses in Consecutive CT Examinations from Five University of California Medical Centers. Radiology 2015; 277: 134-141

[69] Radiology ESo. Summary of the European Directive 2013/59/Euratom: essentials for health professionals in radiology. Insights into imaging 2015; 6: $411-417$ 
